\title{
Supply Chain Decisions of Fresh Products for Competitive Suppliers with Traceability Technology
}

\author{
Qi Zheng*1,a , Qian Xiao², Peiting Zhao ${ }^{3}$ \\ ${ }^{1}$ School of Management Shanghai University of Engineering Science Shanghai, China \\ ${ }^{2}$ School of Management Shanghai University of Engineering Science Shanghai, China \\ ${ }^{3}$ School of Management Shanghai University of Engineering Science Shanghai, China
}

\begin{abstract}
This paper focuses on the impact of traceability technology adoption on supply chain coordination. We consider a fresh product supply chain consisting of two suppliers and one retailer with centralized and decentralized decision-making. Considering the factors of the tag cost of traceability technology and the freshness of the product, two scenarios-with and without traceability technology are analyzed. The mathematical model is applied to investigate the impact of applying traceability technology on decision-making and profit of supply chain when two suppliers compete. The results show that: (1) the fresh product supply chain with the traceability technology is more profitable than the case that without the traceability technology; (2) when the tag cost of the traceability technology is within the threshold, the supplier's profit decreases with the increase of the tag cost, and it is always greater than the corresponding profit when comparing with the case that without adopting the traceability technology; (3) if the tag cost of the traceability technology is too high, the retailer can use cost sharing or bargaining with the supplier to encourage him to implement the technology.
\end{abstract}

\section{Introduction}

Fresh produce is necessary in people's daily lives and is also an essential part of the food industry. However, as sellers of special perishable product, grocery retailers often suffer massive losses due to damage and spoilage of fresh produce [1]. In Europe, the attrition rate of fresh produce in retail stores is as high as $15 \%$, causing billions of dollars in losses each year [2]. In the USA, the losses in the fresh produce industry are up to $\$ 30$ billion annually [3]. In China, more than $25 \%$ of fresh produce decays during circulation, which causes a loss of more than $¥ 150$ billion every year [4]. Hence, massive losses of fresh produce have become a critical issue that many retailers must confront.

Where do these losses of the fresh products arise from? According to the operations processes of the fresh produce supply chain, the losses mainly arise from transportation, delivery and retail. During fresh produce transportation and delivery to the grocery, if distribution is inadequately handled, such as keeping fresh produce at improper places and temperatures on the shelves, spoilage will increase greatly. Additionally, if fresh produce is misplaced during retailing, consumer buying choices will be affected, and the losses of fresh produce will also increase.

How can the loss rate of produce be reduced? The most effective solution is traceability technology implementation. traceability technology is considered an effective way to solve the problem of misplacement because it enables the tracking of produce and access to real-time inventory information [5]. In addition, the retailer will not miss sales opportunities since the application of traceability technology makes replenishment more timely [6]. However, traceability technology implementation will influence the benefits and costs of the different members of FPSCs. If there is a lack of effective revenue-sharing and cost-sharing among the members of the supply chain, traceability technology implementation will lead to inefficient cooperation in the FPSC, which will greatly aggravate the losses of fresh produce. Therefore, an increasing number of researchers and practitioners are paying attention to the application of traceability technology.

In the FPSC management with competitive suppliers, the application of traceability technology increases costs to the suppliers, and reduces the misplacement rate of the retailer. Free-rider behavior by the retailer causes him to share in the benefits of the supplier and affects the operation of the FPSC. Therefore, how to coordinate the FPSC in centralized and decentralized decisions with traceability technology has become a question worth considering. Another major challenge is to maintain a low loss rate for fresh produce. Traceability technology has been verified to significantly improve efficiency in different stages of the supply chain, and the related effects on costs are by no means negligible [7].

Therefore, we consider a FPSC of two competitive suppliers with centralized and decentralized decisionmaking, and investigate the impact of the tag cost of

\footnotetext{
a* Corresponding author: zhengqi@sues.edu.cn
} 
traceability technology and the freshness of the product on the profit of the FPSC under two scenarios-with and without traceability technology. The supply chain will obtain more profit after adopting traceability technology. This is a worthy scientific problem for study.

\section{Problem Desciption}

We consider a two-tier FPSC composed of two suppliers and one retailer. In this chain, the retailer purchases two single-cycle fresh produce from two suppliers simultaneously. The retailer and the supplier act as a Stackelberg leader and a follower, respectively. In this paper, we examine the impact of the application of traceability technology on circulation efficiency and decision-making in supply chains. There are three scenarios about the supplier's investment of traceability technology: two suppliers do not implement traceability $(S S)$; one supplier implements traceability technology and the other does not $(\bar{S} S)$; and two suppliers implement traceability technology $(\overline{S S})$.

Based on the market demand for the fresh product, the retailer determines its ordering quantity before the product's selling season, the suppliers determine their own wholesale price, and then, the product is transported by the supplier and arrives at the target market when the product's selling season begins. Finally, the consumers obtain the fresh produce at a certain selling price which are determined by the retailer.

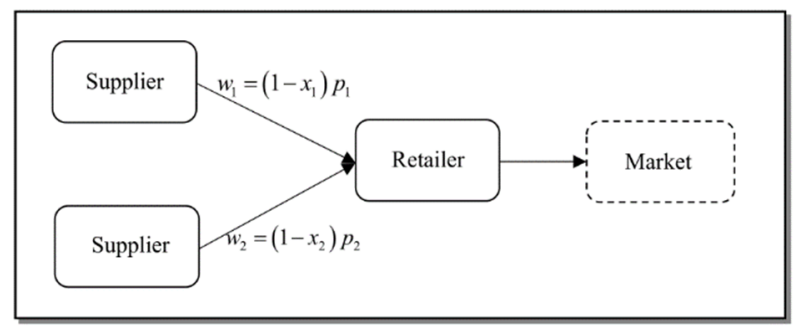

Fig 1. Research Framework in the FPSC

To simplify the mathematical model without losing generality, we assume the following:

- The salvage value of the fresh product at the end of the sale period is zero.

- The supplier and retailer are risk-neutral; thus, they always pursue maximum profit.

- A stock-out is not allowed.

Before formulating the supply chain decision problem with traceability technology, we introduce the following notations.

Table1. Notation for mathematical models

\begin{tabular}{|c|l|}
\hline Parameters & \multicolumn{1}{c|}{ Brief description } \\
\hline$c$ & The production cost of fresh produce \\
\hline$u_{i}$ & The retailer's demand for products $i$ \\
\hline$Q_{0}$ & $\begin{array}{l}\text { The retailer's demand when the selling price is } \\
\text { zero }\end{array}$ \\
\hline$\alpha$ & The price elasticity of demand \\
\hline
\end{tabular}

\begin{tabular}{|c|l|}
\hline Parameters & \multicolumn{1}{c|}{ Brief description } \\
\hline$\beta$ & The preference of the supplier \\
\hline$\theta(t)$ & The produce's freshness \\
\hline$k$ & $\begin{array}{l}\text { The coefficient of competition between two } \\
\text { suppliers }\end{array}$ \\
\hline$w_{i}$ & The supplier's wholesale price for product $i$ \\
\hline$p_{i}$ & The retailer's selling price for product $i$ \\
\hline$\lambda$ & The ratio of misplaced quantity to inventory \\
\hline$\varphi$ & The ratio of produce's loss to inventory \\
\hline$\delta$ & $\begin{array}{l}\text { The proportion of the retailer's tag cost } \\
\text { investment }\end{array}$ \\
\hline$S$ & The salvage of misplaced produce \\
\hline$x_{i}$ & The ability of the retailer's bargaining power \\
\hline$\Pi$ & The profit \\
\hline
\end{tabular}

We develop a mathematical model for an FPSC with traceability technology. The fresh produce's demand function

is: $u_{i}=Q_{0}-\alpha p_{i}+k_{1}\left(p_{j}-p_{i}\right)+\beta\left(\theta\left(t_{i}\right)-k_{2}\left(\theta\left(t_{j}\right)-\theta\left(t_{i}\right)\right)\right) \cdot$ In order to simplify the calculation, we assume $k=k_{1}=k_{2}$, $\vartheta_{i}=\theta\left(t_{i}\right)-k\left(\theta\left(t_{j}\right)-\theta\left(t_{i}\right)\right)$. Therefore, the demand function is $u_{i}=Q_{0}-\alpha p_{i}+k\left(p_{j}-p_{i}\right)+\beta \vartheta_{i}$. Here, the demand is inversely proportional to the selling price, and it is affected by the differentiation degree of the products. When the retailer's selling price is unpredictable, the supplier determines the wholesale price through bargaining game. Therefore, the optimal selling price determined by the retailer must meet the principle of profit maximization. The bargaining process between the supplier $i$ and the retailer $i$ is as follows: $w_{i}=\left(1-x_{i}\right) p_{i}, p_{i}=\arg \max \prod_{R}$, $i=1$, where $x_{i}$ is the bargaining power of the retailer in the asymmetric situation, $0<x_{i}<1, c_{i}<w_{i}<p_{i}$.

The retailer orders the fresh products from the supplier according to the predicted market demand, and neither of them can share inventory information. There are two major problems in retailer's inventory: misplacement and loss. The ratio of the misplacement to the inventory is $\lambda(\lambda \in[0,1])$, the ratio of the loss to the inventory is $\varphi(\varphi \in[0,1])$. When the inventory is $Q$, the quantity of the products that can be sold normally is $(1-\lambda-\varphi) Q$, the remaining fresh product is $\lambda Q$. The unit salvage that they have at the end of the sales period is $S$.

In reality, we can use the traceability technology to realize real-time monitoring to solve the problem of the misplacement and loss of fresh products. The traceability technology is often adopted by the supplier. Therefore, it is assumed that the supplier bears the cost the traceability technology. In this paper, we only consider the variable cost of the investment on the traceability technology, and the fixed investment cost is not considered. 


\section{A Coordination Model Under Three Scenarios}

In this section, we assume a single-period setting. The product is perishable and, hence, must be salvaged at the end of the period. We consider three structures in the mathematical model, which is $S S$ model, $\bar{S} S$ model and $\overline{S S}$ model. Here, $S S$ represents the case that two suppliers do not adopt the traceability technology, $\bar{S} S$ represents the case that one supplier adopts the traceability technology, $\overline{S S}$ represents the case that two suppliers adopt the traceability technology.

\section{1 $S S$ model}

When the retailer is the leader, the suppliers do not adopt the traceability technology. The retailer's misplaced inventory quantity is $\lambda u$, the loss quantity of the fresh produce is $\varphi u$, and the inventory available quantity is $(1-\lambda-\varphi) u$. Thus, the retailer's profit is

$$
\Pi_{R}^{S S}(p)=\sum_{i=1}^{2}\left\{\left[(1-\lambda-\varphi) p_{i}+\lambda s_{i}\right] u_{i}-w_{i} u_{i}\right\}
$$

Taking the second derivative of Eq.(1) with respect to the selling price, we have

$$
\frac{\partial^{2} \Pi_{R}^{S S}(p)}{\partial p_{i}^{2}}=-2(\alpha+k)(1-\lambda-\varphi)<0
$$

From the equation, we know that the retailer's profit function is strictly concave in its selling price. Thus, there exists a maximum value.

Taking the first derivative of Eq.(1) to the selling price, then we set it equal to 0 . Therefore, the selling price of the retailer is

$$
p_{i}^{S S}=\frac{\left[k^{2}-(\alpha+k)^{2}\right]\left(\lambda s_{i}-w_{i}\right)+(\alpha+2 k)(1-\lambda-\varphi)\left(Q_{0}+\beta \vartheta_{i}\right)}{2(1-\lambda-\varphi)\left[(\alpha+k)^{2}-k^{2}\right]}
$$

According to the bargaining power of the retailer, we can get the wholesale price of the supplier

$$
w_{i}=\left(1-x_{i}\right) p_{i}
$$

Substituting Eq.(4) into $p_{i}^{S S}$, we get the optimal selling price of the retailer

$$
p_{i}^{S S^{*}}=\frac{(1-\lambda-\varphi)\left(Q_{0}+\beta \vartheta_{i}\right)-\alpha \lambda s_{i}}{\left[1+x_{i}-2(\lambda+\varphi)\right] \alpha}
$$

Then, we can derive the optimal wholesale price of the supplier

$$
w_{i}^{S S^{*}}=\left(1-x_{i}\right) \frac{(1-\lambda-\varphi)\left(Q_{0}+\beta \vartheta_{i}\right)-\alpha \lambda s_{i}}{\left[1+x_{i}-2(\lambda+\varphi)\right] \alpha}
$$

The fresh produce's optimal demand is:

$$
u_{i}^{S S^{*}}=\frac{(\alpha+k) \lambda s_{i}-k \lambda s_{j}+\left(x_{i}-\lambda-\varphi\right)\left(Q_{0}+\beta \vartheta_{i}\right)}{1+x_{i}-2(\lambda+\varphi)}
$$

Therefore, the retailer's optimal profit is

$\Pi_{R}^{S S^{*}}(p)=\sum_{i=1}^{2}\left\{(1-\lambda-\varphi) \frac{\alpha \lambda s_{i}+\left(x_{i}-\lambda-\varphi\right)\left(Q_{0}+\beta \vartheta_{i}\right)+k \lambda\left(s_{i}-s_{j}\right)}{\alpha\left[1+x_{i}-2(\lambda+\varphi)\right]^{2}}\right\}$

Because the supplier's profit is $\Pi_{S i}^{S S}(w)=u_{i}\left(w_{i}-c_{i}\right), i=1,2$. Substituting Eq.(6) and
Eq.(7) into the supplier's profit function, we obtain the supplier's optimal profit

$$
\Pi_{S i}^{S S *}=\frac{\left(1-x_{i}\right)\left[(1-\lambda-\varphi)\left(Q_{0}+\beta \vartheta_{i}\right)-\alpha \lambda s_{i}\right]}{\alpha\left[1+x_{i}-2(\lambda+\varphi)\right]^{2}}
$$

\section{$3.2 \bar{S} S$ model}

When the supplier $j$ adopts the traceability technology, the fresh produce obtained from the supplier $j$ will not be misplaced or damaged due to the free-rider effect for the retailer. However, the inventory of the fresh produce obtained from the supplier $i$ is still inaccurate. Therefore, the retailer's profit is

$$
\Pi_{R}^{\bar{S} S}(p)=u_{j}\left(p_{j}-w_{j}\right)+\left[(1-\lambda-\varphi) p_{i}+\lambda s_{i}\right] u_{i}-w_{i} u_{i}
$$

Similarly, the wholesale price of the supplier is

$$
w_{j}^{\overline{S S}}=\left(1-x_{j}\right) p_{j}
$$

The fresh produce's demand is

$$
u_{j}^{\bar{S} S}=Q_{0}-\alpha p_{j}+k\left(p_{i}-p_{j}\right)+\beta \vartheta_{j}
$$

Taking the second derivative of Eq.(10) with respect to the selling price, we have $\frac{\partial^{2} \Pi_{R}^{\overline{S S}}(p)}{\partial p_{i}^{2}}<0, \frac{\partial^{2} \Pi_{R}^{\overline{S S}}(p)}{\partial p_{j}^{2}}<0$.

From the equation, we know that the retailer's profit function is strictly concave in its selling price. Thus, there exists a maximum value.

Taking the first derivative of Eq.(10) to the selling price, then we have

$$
\begin{gathered}
p_{j}^{\bar{S} *^{*}}=\frac{\left(Q_{0}+\beta \vartheta_{j}\right)+k(\alpha+k) \lambda s_{i}(\Phi-1)}{\left(1+x_{j}\right)\left(2 \Phi-1+x_{i}\right)(\alpha+k)^{2}-k^{2}\left(x_{j}+\Phi\right)\left(\Phi+x_{i}\right)} \\
p_{i}^{\bar{S} s^{*}}=\frac{\lambda s_{i}\left[k^{2}\left(x_{j}+\Phi\right)-\left(1+x_{j}\right)(\alpha+k)^{2}\right]}{\left(1+x_{j}\right)\left(2 \Phi-1+x_{i}\right)(\alpha+k)^{2}-k^{2}\left(x_{j}+\Phi\right)\left(\Phi+x_{i}\right)}
\end{gathered}
$$

Where $\Phi=1-\lambda-\varphi$

The fresh produce's demand for supplier $j$ is

$u_{j}^{\bar{S} S}=\left(Q_{0}+\beta \vartheta_{j}\right)-\frac{k\left(Q_{0}+\beta \vartheta_{j}\right)\left[k\left(x_{j}+\Phi\right)+(\alpha+k)\left(x_{j}+1\right) \Phi\right]}{K}$

The fresh produce's demand for supplier $i$ is

$$
u_{i}^{\bar{S} S}=\frac{k\left(Q+\beta \vartheta_{i}\right)\left[(\alpha+k)\left(1-x_{i}-2 \Phi\right)-k \Phi\left(x_{i}+\Phi\right)\right]}{K}
$$

Substituting Eq.(15) and Eq.(16) into Eq.(10), we can derive the retailer's optimal profit.

The profit of the supplier $j$ is

$$
\Pi_{S j}^{S S}(w)=u_{j}\left(w_{j}-c_{j}-t\right)
$$

Substituting Eq.(11), Eq.(13) and Eq.(15) into Eq.(17), we can derive the optimal profit $\prod_{S j}^{\bar{S} S^{*}}$.

The profit of the supplier $i$ is

$$
\Pi_{S i}^{\overline{S S}}(w)=u_{i}\left(w_{i}-c_{i}\right)
$$

Substituting Eq.(14) and Eq.(16) into Eq.(18), we can derive the optimal profit $\prod_{S i}^{\overline{S S}}$. 


\section{3 $\overline{S S}$ model}

When two suppliers adopt the traceability technology, the fresh produce will not be misplaced or damaged. Therefore, the retailer's profit is

$$
\Pi_{R}^{\overline{S S}}(p)=\sum_{i=1}^{2}\left(u_{i} p_{i}-w_{i} u_{i}\right)
$$

When the retailer's bargaining power is $x$, the wholesale price is

$$
w_{i}^{\overline{S S}}=\left(1-x_{i}\right) p_{i}, i=1,2
$$

Similarly, taking the second derivative of Eq.(19) with respect to the selling price $\overline{p_{i}}$, we have $\frac{\partial^{2} \Pi_{R}^{\overline{S S}}(p)}{\partial p_{i}^{2}}<0$.

From the equation, we know that the retailer's profit function is strictly concave in its selling price. Thus, there exists a maximum value.

Taking the first derivative of Eq.(19) to the selling price, then we have the equilibrium solutions

$$
\begin{array}{r}
p_{i}^{\overline{S S} *}=\frac{Q_{0}+\beta \vartheta_{i}}{\left(1+x_{i}\right) \alpha}, i=1,2 \\
w_{i}^{\overline{S S^{*}}}=\frac{\left(1-x_{i}\right)\left(Q_{0}+\beta \vartheta_{i}\right)}{\left(1+x_{i}\right) \alpha}, i=1,2 \\
u_{i}^{\overline{S S} *}=\left(Q_{0}+\beta \vartheta_{i}\right)\left[1-\frac{\alpha+k}{\left(1+x_{i}\right) \alpha}+\frac{k}{\left(1+x_{i}\right) \alpha}\right]
\end{array}
$$

Therefore, the retailer's optimal profit is

$$
\Pi_{R}^{\overline{S S} *}=\sum_{i=1}^{2} \frac{x_{i}\left(Q_{0}+\beta \vartheta_{i}\right)^{2}}{\left(1+x_{i}\right) \alpha}\left[1-\frac{\alpha+k}{\left(1+x_{i}\right) \alpha}+\frac{k}{\left(1+x_{j}\right) \alpha}\right]
$$

$$
\begin{aligned}
& \text { The supplier's profit } \\
& \Pi_{S i}^{\overline{S S}}(w)=u i(w i-c i-t), i=1,2 \\
& \text { Substituting Eq.(22) and Eq.(23) into the supplier's } \\
& \text { profit function, we can derive the supplier's optimal profit. } \\
& \prod_{S i}^{\overline{S S} *}=\left(Q_{0}+\beta \vartheta_{i}\right)\left[\frac{\left(1-x_{i}\right)\left(Q_{0}+\beta \vartheta_{i}\right)}{\left(1+x_{i}\right) \alpha}-c_{i}-t\right]
\end{aligned}
$$

\section{Comparative Analysis Under Different Structure Moderls}

To reveal how traceability technology affects the selling price, the wholesale price and the profit under different structure models, in this section, we compare the $\overline{S S}$ model and $\bar{S} S$ model.

Proposition 1. In the $\overline{S S}$ model, the tag cost of traceability technology has no correlation with the selling price, the wholesale price and the market demand. However, through changing the retailer's inventory availability, the retailer's profit can be indirectly affected and the profit of the supplier who invests in the traceability technology can be directly affected.
In the $\overline{S S}$ model, $p_{i}^{\overline{S S} *}=\frac{\left(Q_{0}+\beta \vartheta_{i}\right)}{(1+x) \alpha}, i=1,2$,

$u_{i}^{\overline{M M}}=\left(Q_{0}+\beta \vartheta_{i}\right)\left[1-\frac{\alpha+k}{(1+x) \alpha}+\frac{k}{(1+x) \alpha}\right], i=1,2 ; j=3-i$

$w_{i}^{\overline{S S}^{*}}=\frac{\left(1-x_{i}\right)\left(Q_{0}+\beta \vartheta_{i}\right)}{\left(1+x_{i}\right) \alpha}, i=1,2$.

From the equation, we can see that the tag cost of traceability technology has no correlation with the selling price, the wholesale price and the market demand.

When the traceability technology is adopted, the retailer's inventory availability is 1. The retailer's inventory availability is less than 1 without traceability technology. Therefore, the retailer's profit can be indirectly affected by the retailer's inventory availability. The supplier's profit is $\Pi_{S i}^{\overline{S S}}(w)=u_{i}\left(w_{i}-c_{i}-t\right), i=1,2$. From the equation, we can see that there is a correlation between the supplier's profit and the tag cost.

Proposition 2. In the $\bar{S} S$ model, the tag threshold that the supplier $j$ is willing to invest in traceability technology

$$
\begin{aligned}
& t_{1}=\frac{(\alpha+k) H+p_{j}^{\overline{S S}}\left(1-x_{j}\right)\left[\left(Q_{0}+\beta \vartheta_{j}\right)+k p_{i}^{\bar{M} M}\right]}{\left(Q_{0}+\beta \vartheta_{i}\right)-(\alpha+k) p_{j}^{S S}+k p_{i}^{S S}}, \text { where } \\
& H=p_{j}^{\overline{S S}}\left[c_{j}-\left(1-x_{j}\right) p_{j}^{\overline{S S}}\right]-p_{j}^{S S}\left[c_{j}-\left(1-x_{j}\right) p_{j}^{\overline{S S}}\right] . \text { In }
\end{aligned}
$$

the $\overline{S S}$ model, the tag cost threshold that the supplier $i$ is willing to invest in traceability technology is $t_{2}=\frac{\alpha\left(Q_{0}+\beta \vartheta_{i}\right)+\alpha A-k(A-B)}{\alpha\left(Q_{0}+\beta \vartheta_{i}\right)\left[x_{i}(\alpha+k)+x_{j}\left(\alpha x_{i}-k\right)\right]} \quad, \quad$ where $A=\frac{\alpha \lambda s_{i}-\left(Q_{0}+\beta \vartheta_{i}\right)(1-\lambda-\varphi)}{2 \lambda+2 \varphi-x_{i}-1}$ $B=\frac{\alpha \lambda s_{j}-\left(Q_{0}+\beta \vartheta_{j}\right)(1-\lambda-\varphi)}{2 \lambda+2 \varphi-x_{j}-1}$.

In the $\bar{S} S$ model, when the supplier with traceability technology can obtain more profit than the supplier without traceability technology, the supplier $j$ is willing to invest in traceability technology. That is $\Delta \Pi^{\overline{S S}}=\Pi_{S j}^{\overline{S S}}-\Pi_{S j}^{S S}>0$. Therefore, we can obtain the tag cost threshold $t_{1}$ for the supplier $j$. Similarly, we can derive the tag cost threshold $t_{2}$ for the supplier $i$.

Proposition 3. When the tag cost of the traceability technology is within the threshold, the supplier's profit decreases with the increase of the tag cost in the $\overline{S S}$ and $\bar{S} S$ model, and it is always greater than the corresponding profit when comparing with the SS model.

From Eq.(17) and Eq.(25), it can be seen that the supplier's profit decreases with the increase of the tag cost. From Proposition 2, we can know that when the tag cost of the traceability technology is within the threshold, $\Delta \Pi^{\overline{S S}}=\Pi_{S j}^{\overline{S S}}-\Pi_{S j}^{S S}>0, \Delta \Pi^{\overline{S S}}=\Pi_{S i}^{\overline{S S}}-\Pi_{S i}^{S S}>0$, that is, $\Pi_{S j}^{\overline{S S}}>\Pi_{S j}^{S S}, \Pi_{S i}^{\overline{S S}}>\Pi_{S i}^{S S}$. Therefore, the supplier's profit with the traceability technology is greater than the case that without the traceability technology. 
Proposition 4. The retailer's profit will increase after adopting the traceability technology. When the initial tag cost of the traceability technology is too high, the retailer can use cost sharing or bargaining with the supplier to encourage him to implement the technology.

The free rider effect of the retailer makes him share the traceability technology that implemented by the suppliers, and helps himself solve the problem of inaccurate inventory. Thus, the increase of the retailer's profit is due to the decrease of inventory loss and products' misplacement. The increased profit is $\Delta \Pi_{R}=(\lambda u s+\varphi u)\left(p_{i}-w_{i}\right)$. The supplier bears the cost of the new technology alone. Therefore, when the tag cost of the traceability technology is higher or exceeds the threshold, the retailer can share the cost with the supplier to encourage the application of the traceability technology.

We assume that the retailer's cost sharing ratio of the new technology is $\delta$, the supplier's cost sharing ratio of the new technology is $(1-\delta) / 2$. Thus, the retailer's profit is $\bigsqcup_{R}^{\overline{S S}}(p)=\sum_{j=1}^{2}\left(u_{i} p_{i}-w_{i} u_{i}-\delta t_{2}\right)$. The supplier's profit is $\amalg_{S i}^{\overline{S S}}(w)=u_{i}\left(w_{i}-c_{i}-\frac{1-\delta}{2} t_{2}\right) \cdot$

From the above discussion, we derive some instructions from the model. In the real life, it is significant to promote the supplier and the retailer to implement the traceability technology. Furthermore, if the tag cost of the traceability technology is too high, then they can share the cost of the new technology to obtain more profit and achieve a win-win result.

\section{Numerical Examples}

In this section, we present some numerical examples to illustrate the theoretical outcomes obtained in the previous sections. Here, we assume that $c_{1}=30, c_{2}=30$, $Q_{0}=300, \alpha=2, k=1, x_{1}=0.3, x_{2}=0.3, \lambda=0.1$, $s_{1}=1, s_{2}=1, \vartheta_{1}=0.7, \vartheta_{2}=0.7$. Using the parameters, we apply them to the previously analysed scenarios. Next, we analyse how the traceability technology impacts the profits of both the suppliers and the retailer.

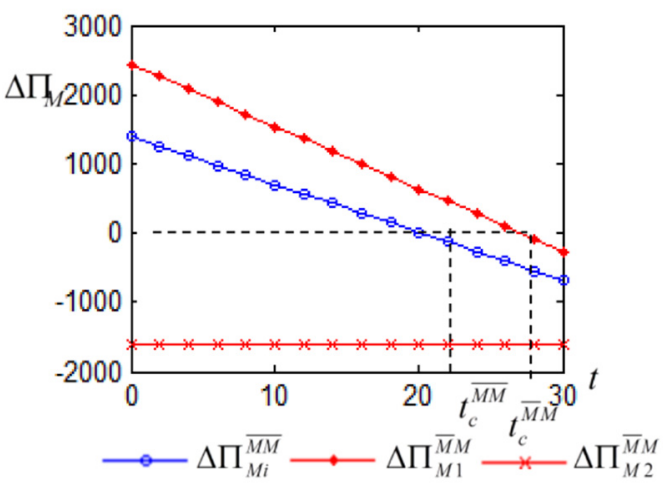

Fig 2. The suppliers' profit difference with the change of the tag cost
From Fig.2, we can see that the threshold of the traceability technology's tag cost that the suppliers can afford in the $\overline{S S}$ model is $t_{c}^{\bar{S} S} \in(0,27.2)$. The threshold in the $\overline{S S}$ model is $t_{c}^{\overline{S S}} \in(0,20.2)$, and $t_{c}^{\overline{S S}}>t_{c}^{\overline{S S}}$. The difference of the supplier's profit decreases with the increase of the tag cost, and $\Delta \Pi_{S 1}^{\overline{S S}}>\Delta \Pi_{S i}^{S S}>\Delta \Pi_{S 2}^{\overline{S S}}$. This shows that in the two competing suppliers, supplier 1 gives priority to the traceability technology, which can enjoy the benefits brought by the technology, and the tag cost he can bear is also higher. However, the profit difference of the supplier 2 decreases to a negative value. When the supplier 2 realizes the threat and then follows the competitor to adopts the traceability technology, the profit will increase. Then, the profit of the supplier 1 decreases, and the tag cost that supplier 1 can bear becomes lower.

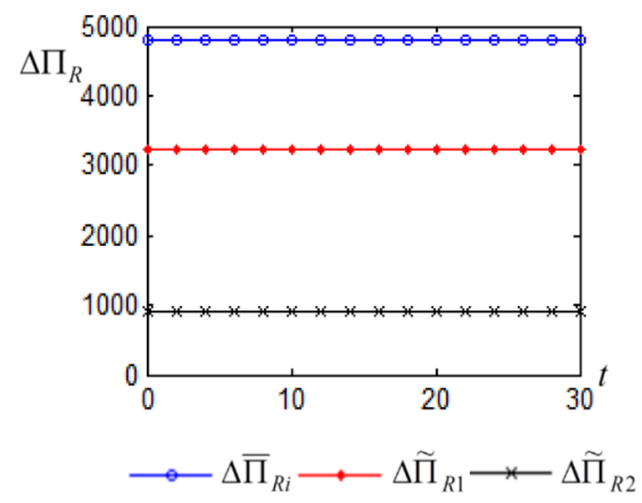

Fig 3. The retailer's profit difference with the change of the tag cost

The results in Fig.3, showing the retailer's profit has no correlation with the tag cost in the three structure models, and the retailer's profit is $\Pi_{R}^{\overline{S S}}>\Pi_{R}^{\overline{S S}}>\Pi_{R}^{S S}$. When the supplier adopts the traceability technology, the retailer can share the spill over effect of the technology for free. When the two suppliers adopt the new technology, the profit is the highest, but the critical cost that the supplier can bear is limited. Therefore, the retailer can adjust the bargaining range and cost sharing contract to coordinate the benefits of both sides, and encourage the suppliers to apply the traceability technology.

Here, we assume that $x_{1}=0.3, x_{2}=0.3$. Thus, we can get the profit of the suppliers and the retailer.

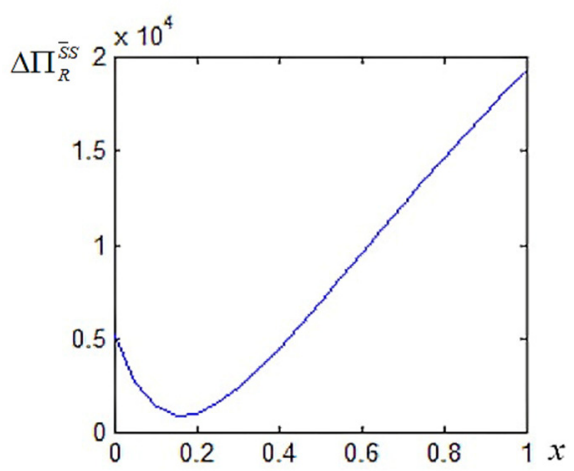

Fig 4. The impact of the bargaining level on the retailer's profit difference 
Fig.4 demonstrates the impact of the bargaining level on the retailer's profit difference between the $S S$ model and $\bar{S} S$ model. We can see that no matter how the bargaining level of the retailer is, the retailer's profit always increases, and it is decreasing first and then increasing. Therefore, the application of the traceability technology by the suppliers is always beneficial to the retailer, and the retailer should actively respond and assist the suppliers in adopting new technology.

\section{Conclusions}

In this paper, we have investigated supply chain decisions for fresh products with competitive suppliers after adopting the traceability technology. We focus on the effectiveness of the traceability technology on FPSC when three structures in the mathematical model. Three cases are considered: $S S$ model, $\bar{S} S$ model and $\overline{S S}$ model. In each case, we determine the optimal decisions in FPSC and the threshold of the tag cost when adoption the traceability technology, the range of the tag cost for achieving a win-win situation in FPSC. Then we compare the parameters in the three different scenarios. In practice, it is necessary to encourage the supplier and the retailer to adopt the traceability technology. In addition, if the tag cost of the traceability technology is too high, then they can share the cost to achieve a win-win result and maximize their own profit. This paper provided a decision-making reference for the enterprises.

\section{Acknowledgment}

This work is supported by Shanghai Planning Office of Philosophy and Social Science (2018EGL010).

\section{References}

1. Zheng, Q., Zhou, L., Fan, T., \& Ieromonachou, P. Joint procurement and pricing of fresh produce for multiple retailers with a quantity discount contract. Transportation Research Part E, 2019, 130, 16-36.

2. Fan, T., Xu, C., \& Tao, F. Dynamic pricing and replenishment policy for fresh produce. Computers \& Industrial Engineering, 2020, 139, 106-127.

3. Haijema, R. e, \& Minner, S. Improved ordering of perishables: The value of stock-age information. International Journal of Production Economics, 2019, 209, 316-324.

4. Wang, C., \& Chen, X. Option pricing and coordination in the fresh produce supply chain with portfolio contracts. Annals of Operations Research, 2017, 248(1), 471-491.

5. Zhang, S., Wang, C., Yu, C., \& Ren, Y. Governmental cap regulation and manufacturer's low carbon strategy in a supply chain with different power structures. Computers \& Industrial Engineering, 2019, 134, 27-36.

6. Yang, H., \& Chen, W. Game modes and investment cost locations in radio-frequency identification (RFID) adoption. European Journal of Operational Research, 2020, 286(3), 883-896.

7. Fan, T., Tao, F., Deng, S., \& Li, S. Impact of RFID technology on supply chain decisions with inventory inaccuracies. International Journal of Production Economics, 2015, 159, 117-125. 